\title{
3D Dynamic Visualization of Single Poplar Combined Growth Model with Structural Model
}

\author{
Chun-Hua $\mathrm{Hu}$ \\ College of Information Science and Technology, Nanjing \\ Forestry University, Nanjing, China, 210037 \\ chunhh@163.com \\ Dong Yang \\ College of Information Science and Technology, Nanjing \\ Forestry University, Nanjing, China, 210037
}

\author{
Ping-Ping Li \\ College of Biology and Environment, Nanjing Forestry \\ University, Nanjing, China, 210037 \\ Ting Yun \\ College of Information Science and Technology, Nanjing \\ Forestry University, Nanjing, China, 210037
}

\begin{abstract}
It is important for real tree visualization and $3 D$ reconstruction based on accessibly collected data. Here the poplar tree, belonged to fast growing woods, was taken as the experimental subject due to its extensive use in industrial manufacturing. For further enhancing the plant lifecycle management for poplar monitoring, controlling and physically analyzing anytime, a novel method of constructing quickly and automatically precision tree models from point clouds and digital image was proposed. The growth model and the morphology structure were set up combined digital image processing methods with measurement parameters, while the leaves were reconstructed with NURBS according to $3 D$ points cloud taken by time-of-flight (ToF) camera. The VC++ and OpenGL were adopted to realize these models, and the three-dimensional visualization of single poplar dynamic simulation platform was developed. A large numbers of experiments were carried out. The results validate that the dynamic simulation platform can reconstruct the poplar with poplar age rapidly and effectively.
\end{abstract}

Keywords-Poplar; 3D visualization; Growth model; Structural model

\section{INTRODUCTION}

Three-dimensional visualization of tree is displaying the morphology and growth process of tree using the computer, which provides a visual platform for forestry information construction [1]. In the last few decades, there have been a lot of trees visualization soft wares [1]. These soft wares were developed based on measured data to build tree structure model [2-5], and then according to the structure model, the 3D visualization of trees were realized. The other structure models of trees were a dynamic mathematical model of tree based on the rule of plant knowledge [6, 7]. In addition, 3D visualization of trees was established by combining the dynamic model of trees with the environment growth model, which was the tree structure and function model $[8,9]$.

Many scholars divided the tree into stems, leaves, trunks, and branches respectively. The tree trunks were represented by 3D geometry. Kawaguchi [2] used circular polygons to reconstruct the tree branches, but this method was not good for branches connecting part. Bloomenthal [3] proposed a free surface method connecting link branches smoothly with a more natural description of generalized cylindrical branches. In order to make the tree more realistic, the image acquisition equipment was used to collect the actual trees. Teng et al. [4] reconstructed the tree based on the true picture of trees. First, images of tree were obtained using camera, and then the tree trunks points and leaves collected from these images to construct the tree trunks and leaves. However, this method only visualized leaves as a whole shape. Method of point cloud data to achieve $3 \mathrm{D}$ visualization of trees mainly used laser scanning [5].

Theoretical biologist, Lindenmayer [6], proposed a famous L system based on grammar, which focused on the topology of the plant. Prusinkiewicz [7] proposed string generated by rewriting rules through the method of turtle figure geometric interpretation and the $\mathrm{L}$ system is improved to develop the virtual plant modeling software L-studio, which simulated different types of plants.

Parveaud et al. [8] studied walnut trees to establish 3D visualization model, through measuring number of walnut tree leaves of different ages, different light according to the area of leaves, azimuth angle and blade shape, and reconstructed the walnut trees with ages. Crops such as corn and wheat were established a climate environment illumination and crop growth mechanism model, but for the forest tree, growth and function model is hard to establish. Guo et al. [9] constructed structure and function model at 18 years and 41 year old pines using Greenlab.

In fact, agriculture and forestry urgently need a software to reflect the growth of a plant or tree. For example, the 3D visualization model is established with the forest growth forecast and harvesting theory, which can be used to predict tree growth and harvesting time. Based on the method of image data, it is easy to realize the visualization of the whole tree, but it is difficult to reconstruct the visualization of the tree-leaves. Digital visualization of three-dimensional based on point cloud is time-consuming and not easily realized, especially leaves block each other. Based on the characters of physiological rules, the physiological rules of large trees are difficult to establish. Based on the structure and function of the model, it can well reflect the relationship between tree growth and growth environment, but a complete physiological function model is less. Poplar growth is fast, and measurement of the structural and geometrical parameters is an intricate problem such as the tree height, the tree branch length, the tree branch angle, etc. Many measurement parameters methods were using destructive samplings. However, these destructive samplings are both slow and costly for measuring parameters 
of big tree. There are some problems for 3D visualization measurement of big tree, so this paper presented a novel method for reconstructing poplar growth model and morphology structural model, which combined digital image processing methods with measurement parameters. The leaves were reconstructed with NURBS according to 3D points cloud taken by time-of-flight (ToF) camera. According to these measurement parameters, the growth model of poplar was built, and the dynamic 3D visualization model of poplar under different conditions was established based on the model of poplar and image database.

\section{THE METHOD FRAMEWORK}

In this study, the branch angle model and branch depth model were established. The 3D dynamic visualization poplar platform was set up by combining our previous work with these branch angle model and depth model. The measurement method of the taking data with different ages of poplar height and diameter at breast height (DBH) data was employed to establish the height model and DBH model [10]. In order to effectively obtain branch length of the poplar, a digital image was introduced to build the branch length model [10]. Based on our previous work [10], the branch depth model and branch angel model were established by using digital image processing method. ToF camera was employed to obtain the three-dimension information of leaves, and the 3D leaves of every layer were reconstructed using NURBS. According to the established mathematical model, leaves and stems texture database, a plant poplar dynamic 3D visualization display interface was developed using VC ++ and OpenGL graphics library. In this paper, the dynamic 3D visualization simulation technology of the poplar is shown in Fig. 1.

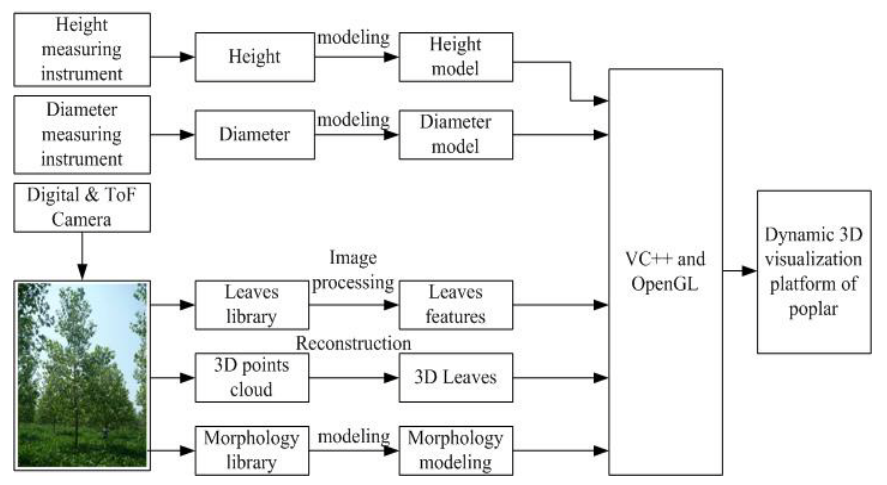

Fig.1 The framework of 3D visualization for poplar

\section{BRANCH ANGLE AND DEPTH MODEL OF POPLAR}

For one year poplar, the angle measuring instrument was used by measuring each branch angle, branch length, and tree height respectively. According to measure a lot of one year poplars, the average branch angle was about 60 degrees, the poplar height was about 2 meters and the average $\mathrm{DBH}$ was $4 \mathrm{~cm}$.

For more than two years and two years of poplar, because the poplar tree is high, the actual measurement way is difficult. In order to obtain the parameters of the structure of poplar tree, the measurement method based on digital image was proposed. As shown in Fig.2, for every branch angle, three points were chosen to calculate the angle between the branch and the trunk. One point $\left(x_{1}, y_{1}\right)$ is one of the branch top measured, the second point $\left(x_{2}, y_{2}\right)$ is one of the branch root and the third point $\left(x_{3}, y_{3}\right)$ is one of the trunk. The branch angle was calculated using the follow formula.

$$
\theta=a \cos d\left(\frac{\left(\operatorname{dot}\left(\left[x_{1}-x_{3}, y_{1}-y_{3}\right],\left[x_{2}-x_{3}, y_{2}-y_{3}\right]\right)\right)}{\operatorname{norm}\left(\left[x_{1}-x_{3}, y_{1}-y_{3}\right]\right) \bullet \operatorname{norm}\left(\left[x_{2}-x_{3}, y_{2}-y_{3}\right]\right.}\right)
$$

Where acosd is inverse cosine function, dot is point product function, and the norm is vector function. According to the formula (1), the branches angles of the trees were calculated from two years and more than two years. As shown in table 1, the average angle of the branches of the 8 years old trees were shown. The first level branch-angle is small, and the second branch-angle began to increase slowly, and the old branch angle is relatively large. This phenomenon may due to the tree growth for a long time, and the branches are thick and have heavier weight, which make the branches bent down. There is also a certain relationship between the angle of the branch and the environment of planting. In other words, the tree branchangle planted in the edge is larger than the one in the middle. As shown in the table 1 , the first level branch-angle is relatively small, less than 20 degrees, the second level average angle is about 23 degree. From the first level to the 8th level, the branch angle is gradually become bigger, to the 8th level, the angle becomes close to 90 degrees and more than 90 degrees.

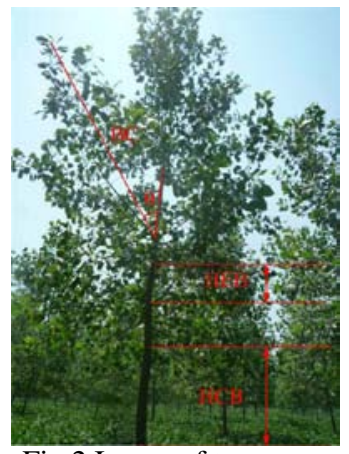

Fig.2 Image of tree

Table1 The average branch angle changes with depth for 8 years old poplar

\begin{tabular}{|c|c|c|c|c|c|c|c|c|}
\hline Branch levels & 1 level & 2 level & 3 level & 4 level & 5 level & 6 level & 7 level & 8 level \\
\hline Average angle & 13.392 & 23.316 & 33.114 & 38.788 & 41.922 & 44.718 & 49.728 & 84.40 \\
\hline
\end{tabular}

A branch depth is also crucial parameters affecting the crown structure. For a big tree, it is difficult to measure the crown depth by manual measurement. So the measurement method combining digital image with measurement instrument was proposed to obtain every branch depth. As shown in Fig.3, for the oldest branch, it is easy to measure the branch height 
using the height measuring instrument, which uses single chip microcomputer as the control core combing sonar sensor with angle sensor. The sonar sensor was utilized to measure the distance from the instrument to the tree, and the angle sensor was utilized to measure the angle between the branches and the instrument. As shown in Fig.3, the height to crown base (HCB) was measured and calculated using the following algorithm.

$$
H C B=d * \operatorname{tg} \beta+b
$$

Where the $d$ is the distance from the instrument to the tree, the $\beta$ is angle between the branches and the instrument, and $b$ is the height from the instrument to the tree root. While the other branch height was calculated by the proportion of tree crown height to branch height in digital image, as shown in Fig.2.

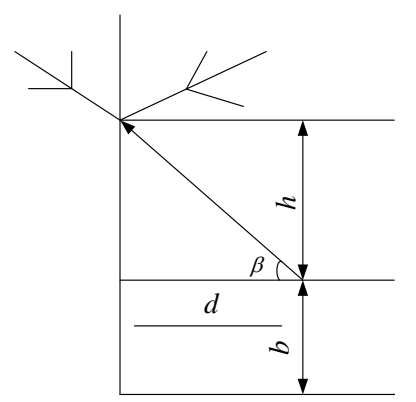

Fig.3 Branch height measurement

\section{3D VISUALIZATION FOR PLANT LEAVES BASED ON NURBS}

In order to obtain the $3 \mathrm{D}$ points of leaves, the time-of-flight (ToF ) camera was utilized to take the 3D point cloud of leaves. Because the 3D point cloud achieved for the whole tree using laser were time-consuming, and the segmentation of leaves was intricate, this paper utilized the ToF camera to obtain 3D points of partly leaves in different direction. In order to obtain the object, the segmentation method based on particle swarm optimization (PSO) in my previous research proposed was employed to segment the depth image. According to the segmented depth image of leaves, the 3D information (X,Y and Z) of a leaf was extracted. The 3D point cloud of a leaf was shown in Fig.4 (a).

Because the NURBS (Non-Uniform Rational B-Splines) method can describe free curve surface and can accurately represent advantages quadratic surface [11]. This paper used it to reconstruct curve surface of the poplar leaves. According to the $3 \mathrm{D}$ point cloud, some control points were chosen, and the NURBS surface was used to reconstruct the curve surface of a leaf. The result of reconstruction for a poplar leaf is shown in Fig.4(b).

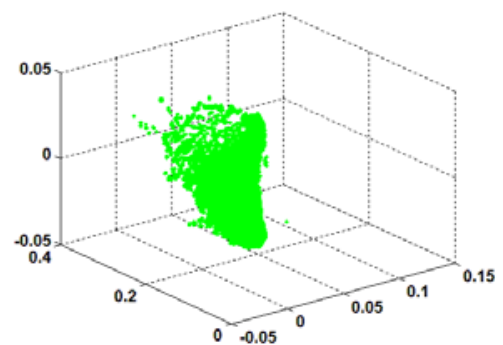

(a)

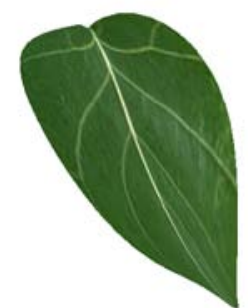

(b)

Fig. 4 The results of a leaf, (a) 3D information of a leaf, (b) 3D reconstruction for poplar leaf based on NURBS

\section{3D VISUALIZATION PLATFORM EXPERIMENTS FOR POPLAR}

The trunk diameter of the tree, in a certain way, is reducing from the root to the top. For 8 years old poplar, the top radius is 0.4 times of the root radius. Every branch of the poplar tree has a self similar characteristic, so the branch uses the self similar characteristic, and the branch direction according to the stochastic growth model through actual measurement. In order to preserve the natural characteristic of branch, the random numbers from -5 degree to +5 degree were added to the stochastic growth model. The leaves are important to reconstruct the virtual tree. Different viewing angles have different visual effects of leaves and the sizes of leaves have also different. There are different sizes for one leaf if it is observed in different visual perception directions. Through a large number of statistical and measurement analysis, for every level branch, the old leaves sizes were smaller than the new leaves, while the tender leaves sizes were smaller than the new leaves. So the visualization for a leaf is complex, including the orientation, the size and the distance to the center of crown. In this paper, the orientation of a leaf was calculated by computing the angle between the normal vector of the leaf and the visual perception direction. A lot of data were collected to simulate the leaves sizes model, and the size model was utilized the $\cos (\theta) * \max \left(L_{s}\right)$, where the $\theta$ is the angle between the normal vector of the leaf and the visual perception direction. The max $L_{s}$ is maximum leaf size in different directions. For the same branch, different level leaf has different size, and the size was decided by the level of leaf. So the level leaf size model is $S=0.6 *\left(0.65+\sin \left(C^{*} \pi / z_{c}\right)\right) * \max \left(L_{s}\right)$, where the $c$ is the level of leaf, and the $z_{c}$ is the total level number of a branch.

According to the poplar growth model [10], the DBH model [10], the branch model, the leaves model and the trunk 
taper, the VC + + and OpenGL graphics library were adopted to recombine a single poplar. In this paper, the cylinder model and the generalized cylinder model were utilized to serve as the trunk model. The trunk of the tree is fitted with a cylinder, as shown in Fig.5. In order to show the nature of the tree, a random value is added to each parameter, and the results are shown in Fig.5. The shape of the tree is typically oval, which is similar to the actual shape of the poplar. Direct cone shows poplar similar morphology, but each branch was not natural, with no distortion. However, the actual tree trunk due to environmental impact, the trunk is not too straight, so there is a certain degree of bending. Based on the basis of the generalized cylinder shape, trunk is divided into four equal parts, each equal parts of the bottom radius were calculated, and the top radius of cylinder has small inclinations. The simulation of the poplar was shown in Fig.6.

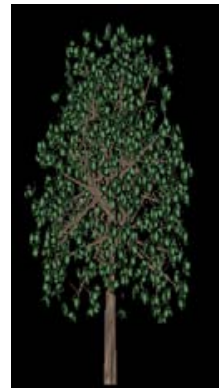

Fig.5 Poplar stem using conical table model

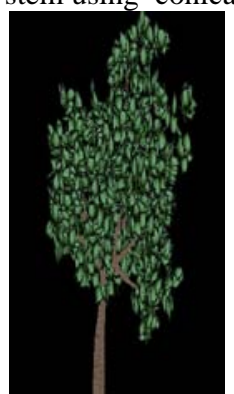

Fig.6 Poplar stem using generalized cylinder model

$\mathrm{VC}++6.0$ and OpenGL were adopted to jointly develop poplar visualization platform with the plant age change. Fig.7 showed a five years old poplar visual interface. From the visualization platform, we can see that the model of poplar growth and morphology structure in this study are more natural and real.

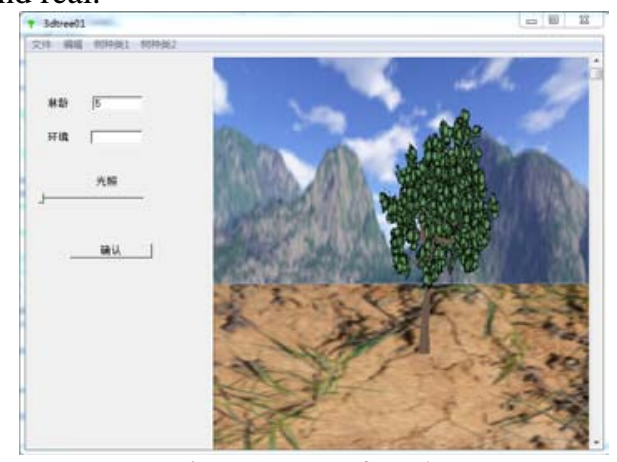

Fig.7 5 years of poplar

To further improve effectiveness of this visualization method for poplar, a large number of statistical analyses of poplar in southern China were carried out. The proposed method in this paper was utilized to establish the poplar growth model and morphology structural model, and the platform for dynamical poplar was set up. The experiments demonstrate that this reconstruction method is faster than that of directly using 3D point cloud, especially for the big tree, and the 3D point cloud can't realize the poplar visualization with the growth model. 50 poplar trees from 1 year to ten years were measured, and the average branch-angles and the variance branch-angles were analyzed respectively. As 8 years old poplars for example, the results between real parameters and the experimental parameters were shown in Fig.8, $\bullet$ presents the errors of measurement, and the $\rightarrow$ * presents the errors of experiments. The average parameters of measurement and experiment had small errors. The results of experiments demonstrated that this method proposed in this paper is effective for reconstructing the $3 \mathrm{D}$ visualization for poplar.

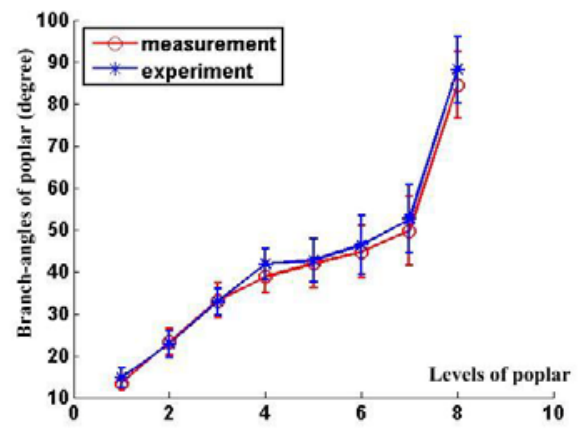

Fig.8 The errors for 8 years old poplar

A large number of statistics for the model of breast diameter at breast height and the depth of the poplar tree were analyzed. 50 sample points were measured respectively, and the confidence level was 95\%. According to the $\mathrm{T}$ distribution table $t_{0.05 / 2}(50)=2.009$, and the calculated value of breast diameter was $t_{d}=0.3725$ and the poplar height calculated value was $t_{h}=1.0325$. The both calculated values are outside the territory rejected. So the DBH growth model and the height growth model are suitable for the growth of poplar tree.

\section{CONCLUSIONS}

In this paper, the three-dimensional visualization of single poplar dynamic simulation platform was developed. The digital image processing method was proposed to extract the key points of branches. According to these points, the angle between two vectors method was used to calculate branch angle, and the depth of branch was measured by a self-made tree height measuring device. The point clouds of leaves in different direction were obtained with ToF camera, the segmentation method based on PSO was employed to segment the leaves, and the leaf key points were extracted. According to these key points, the $3 \mathrm{D}$ visualization of the leaf was realized by NURBS surface method. Combining the previous growth model and DBH model with the 3D leave model and the branch angle model, the dynamic visualized simulation platform was realized by $\mathrm{VC}++$ and OpenGL. A lot of experiments were carried out, and the results demonstrate that 
the poplar morphology and growth models established in this paper can better simulate the growth of poplar morphology and the growth of poplar. The experimental results demonstrate that the platform can effectively and rapidly reflect the growth and morphology structure of poplar.

\section{ACKNOWLEDGMENT}

This work is founded by the National 863 High Technical Program of China (2012AA102002-4), the National Natural Science Foundation of China (31300471), the Priority Academic Program Development of Jiangsu Higher Education Institutions and Jiangsu Government Scholarship for Overseas Studies (JS-2014-013).

\section{REFERENCES}

[1] J.R. Rosell, R. Sanz, "A review of methods and applications of the geometric characterization of tree crops in agricultural activities," Computers and Electronics in Agriculture, vol, 81, pp.124-141, 2012.

[2] K. I. Kawaguch, "A morphological study of the form of nature," In ACM Transactions on Graphics (SIGGRAPH 1982 Conference Proceedings), 1982, pp. 223-232.

[3] J. Bloomenthal, "Modeling the mighty maple," In: SIGGRAPH '85: Proceedings of the 12th annual conference on computer graphics and interactive techniques. ACM Press, New York, 1985, pp. 305-311.
[4] C. H.Teng, Y. S. Chen, “ Image-based tree modeling from a few images with very narrow viewing range,” Vis Comput, vol.25, pp.297-307, 2009.

[5] C. Vegaa, A. Hamrouni , S. E. Mokhtari, "PTrees: A point-based approach to forest tree extraction from lidar data ,'International Journal of Applied Earth Observation and Geoinformation, vol.33, pp.98-108, 2014.

[6] A. Lindenmayer, "Mathematical models for cellular interactions in development," Journal of Theoretical Biology, vol.18, no.3, pp.280-315, 1968.

[7] P. Prusinkiewicz, “ Graphical applications of L-systems ,” Proceedings of Graphics Interface '86 / VisionInterface '86, pp. 247-253, 1986.

[8] C. E. Parveaud, J. Chopard, J. Dauzat, etal. "Modelling foliage characteristics in 3D tree crowns: influence on light interception and leaf irradiance,” Trees , vol.22, pp.87-104, 2008.

[9] H. GUO , X D. LEI and L. Veronique, "A functional-structural model for adults of Pinus tabulaeformis based on GreenLab ," Chinese Journal of Plant Ecology, vol.35, no. 4, pp. 422-430, 2011.

[10] C. H. HU, P. P. LI, "Poplar Branch and Trunk Modeling Based on Levenberg-Marquardt," Journal of Agricultural Machinery, vol. 45, no.10, pp.272-276, 2014.

[11] H. Ghasemi, R. Brighenti, X. Y. Zhuang, et al. " Optimization of fiber distribution in fiber reinforced composite by using NURBS functions," Computational Materials Science, vol. 283, pp.463-473, 2014. 